\title{
Avaliação de 100 pacientes com nefrite lúpica acompanhados por dois anos
}

\author{
Analysis of 100 patients with lupus \\ nephritis followed up for 2 years
}

Ana Karla Guedes de Melo ${ }^{(1)}$, Alessandra Barbosa Avelar ${ }^{(2)}$, Flávia Kamy Marciel Maegawa ${ }^{(3)}$, Branca Dias Batista de Souza ${ }^{(4)}$

\section{RESUMO}

Objetivos: Determinar a frequência de remissão total e parcial no tratamento da nefrite lúpica aos 12 e 24 meses de seguimento. Comparar esses subgrupos aos 12 meses, correlacionando as variáveis renais iniciais com a resposta ao tratamento. Analisar e comparar os resultados terapêuticos do subgrupo com glomerulonefrite proliferativa difusa por correlação clínico-patológica ("classe IV clínica") com aqueles de "classe IV histológica", isto é, com biópsia renal comprovada pela Organização Mundial da Saúde. Material e métodos: Foram estudados 100 pacientes consecutivos com diagnóstico de lúpus eritematoso sistêmico (LES) e nefrite, atendidos no Serviço de Reumatologia da Santa Casa de Misericórdia de São Paulo e acompanhados por dois anos. Os portadores de comorbidades que comprometem os rins foram excluídos. Foram analisadas as variáveis demográficas, clínicas, laboratoriais e o índice de atividade da doença (SLEDAI). Os pacientes com classe histológica III, IV ou V receberam corticosteroide e ciclofosfamida como tratamento de indução da nefrite lúpica e aqueles com classe II receberam apenas corticosteroide. Resultados: A idade média ao diagnóstico de LES foi de 24,71 \pm 10,14 anos, com predomínio do sexo feminino (88\%). O SLEDAI calculado ao diagnóstico foi de $16,09 \pm$ 6,48 . Em relação às variáveis renais iniciais, a creatinina média foi de $1,02 \pm 0,49 \mathrm{mg} / \mathrm{dL}$, a proteinúria de 24 horas média foi de 2,57 $\pm 2,39$ g e o anticorpo anti-dsDNA foi encontrado em $66 \%$ dos casos. Todos os pacientes receberam corticosteroide e $75 \%$ utilizaram a ciclofosfamida. Cinquenta e seis pacientes foram submetidos à biópsia renal. Os subtipos II e IV foram os mais prevalentes $(33,9 \%$ e $32,2 \%$, respec-

\section{ABSTRACT}

Objectives: To assess the frequency of total and partial remission in the treatment of lupus nephritis at 12 months and 24 months. To compare these subgroups at 12 months and correlate the initial renal variables with the therapeutic response. To analyze and to compare the therapeutic results of the subgroup with diffuse proliferative glomerulonephritis by clinicopathological correlation ("clinical class $I V$ ") with those with biopsy-proven WHO class IV ("histological class $I V$ "). Patients and methods: One hundred consecutive patients with diagnosis of systemic lupus erythematosus (SLE) and nephritis who attended to the department of rheumatology of a tertiary referral center were studied. The length of the follow up was 2 years. Patients with comorbidities that compromise the kidneys had been excluded. The demographic, clinical and laboratory variables and the disease activity index (SLEDAI) were analyzed. Patients with lupus nephritis WHO class III, IV or V received glucocorticoid and cyclophosphamide for induction of remission and those with class II received only glucocorticoid. Results: The average age at SLE diagnosis was of $24.71+10.14$ years, with a predominance of female gender (88\%). The initial SLEDAI was $16.09 \pm 6.48$. At the time of the diagnosis of nephritis, mean serum creatinine was $1.02 \pm 0.49 \mathrm{mg} /$ $d L$, mean 24-hour urinary protein level was of $2.57 \pm 2.39 \mathrm{~g}$ and the antibody anti-dsDNA was found in $66 \%$ of the cases. All the patients had received glucocorticoids and $75 \%$ had used cyclophosphamide. Fifty-six patients had been submitted to renal biopsy. The most prevalent subtypes were class II and IV (33.9\% and 32.2\%, respectively).

Recebido em (Received on) 01/11/2007. Aprovado (Approved), após revisão, em 21/10/08. Declaramos a inexistência de conflitos de interesse (We declare no conflict of interest).

Trabalho desenvolvido no Departamento de Medicina da Irmandade da Santa Casa de Misericórdia de São Paulo - SP

Study carried out at Department of Medicine of Irmandade da Santa Casa de Misericórdia de São Paulo-SP

1. Médica especialista em Reumatologia e pós-graduanda da Faculdade de Ciências Médicas da Santa Casa de Misericórdia de São Paulo-SP

1. Rheumatologist. Postgraduate student of Medical Sciences School at Santa Casa de Misericórdia de São Paulo-SP

2. Médica especialista em Reumatologia e pós-graduanda da Faculdade de Ciências Médicas da Santa Casa de Misericórdia de São Paulo-SP

2. Rheumatologist. Postgraduate student of Medical Sciences School at Santa Casa de Misericórdia de São Paulo-SP

3. Médica especialista em Reumatologia

3. Rheumatologist

4. Chefe do Serviço de Reumatologia da Irmandade da Santa Casa de Misericórdia de São Paulo e chefe da disciplina de Reumatologia da Faculdade de Ciências Médicas da Santa Casa de Misericórdia de São Paulo - SP

4. Chief of Rheumatology Department at Irmandade da Santa Casa de Misericórdia de São Paulo; Head of the Rheumatology Department of Medical Sciences School at Santa Casa de Misericórdia de São Paulo-SP

Endereço para correspondência (Correspondence to): Branca Dias Batista de Souza - Departamento de Medicina/Reumatologia (Department of Medicine/ Rheumatology). Rua Dr. Cesário Motta Júnior, 112 - Vila Buarque CEP (Zip Code): 01221-000 Tel/Fax: 55 (11) $2176-7281$. 
tivamente). Após 12 meses de acompanhamento, todos os pacientes apresentaram redução significativa da proteinúria de 24 horas, melhora do sedimento urinário e dos valores das frações do complemento $(\mathrm{C} 3$, C4, CH50). A frequência de remissão total aos 12 meses foi 72,7\% e, aos 24 meses, $85,7 \%(p=0,013)$. A remissão parcial ocorreu em 27,3\% dos doentes aos 12 meses e em 14,3\% aos 24 meses. O sexo masculino apresentou menor frequência de remissão total comparado ao feminino aos 12 meses de acompanhamento (45,5\% versus $81,6 \%, p=0,007)$. Dentre as diferentes variáveis estudadas, nenhuma se correlacionou com remissão total ou parcial aos 12 meses. O subgrupo "classe IV clínica" apresentou maior frequência de remissão total que o subgrupo “classe IV histológica". Conclusão: Com o esquema terapêutico usado em nosso serviço, verificou-se um excelente desfecho em dois anos. Não foram observadas correlações entre variáveis clínico-laboratoriais e remissão total ou parcial. O sexo masculino apresentou menores taxas de remissão total comparado ao feminino. Apesar do pequeno número de pacientes estudados e das controvérsias quanto à biópsia renal, a taxa de remissão total foi maior nos pacientes com "classe IV clínica" em relação àqueles com "classe IV histológica".

Palavras-chave: lúpus eritematoso sistêmico, nefrite, remissão total, biópsia renal.

\section{INTRODUÇÃO}

O lúpus eritematoso sistêmico (LES) é uma doença inflamatória crônica, de natureza autoimune, que pode afetar múltiplos sistemas orgânicos. O envolvimento renal no LES é uma causa significativa de morbidade e mortalidade devido à possibilidade de progressão para insuficiência renal e a complicações relacionadas ao tratamento. Pelos critérios do Colégio Americano de Reumatologia (American College of Rheumatology, ACR - 1997), a nefrite lúpica é definida pela presença de proteinúria persistente $(>0,5 \mathrm{~g}$ nas 24 horas) ou maior que $3+$ ou pela cilindrúria (cilindros hemáticos, tubulares, granulosos ou mistos). Entre os padrões histológicos elaborados pela Organização Mundial da Saúde (OMS), a glomerulonefrite proliferativa difusa (classe IV) representa o subtipo mais prevalente e o de pior prognóstico. ${ }^{1}$ A nefrite constitui a principal causa de internações e mortalidade entre os pacientes com LES. ${ }^{2}$

Estudos randomizados anteriores demonstraram que o uso da ciclofosfamida para o tratamento da nefrite lúpica se correlaciona com maiores taxas de preservação da função renal a longo prazo e com menor incidência de doença renal terminal. ${ }^{3}$

Existem controvérsias quanto à indicação de biópsia renal em todos os pacientes com LES e nefrite. Esdaile et al. estabeleceram uma correlação entre os achados clínico-laboratoriais e a classificação histológica da nefrite lúpica, com o intuito de orientar a conduta terapêutica e racionalizar a realização de procedimentos invasivos nessa população ${ }^{4}$ (Tabela 1).
After 12 months, all the patients had significant reduction in the 24hour urinary protein level, improvement in the urinary sediment and increase in the fractional values of the complement (C3, C4, CH50). The frequency of total remission at 12 months was $72.7 \%$ and, at 24 months, $85.7 \%(p=0.013)$. Partial remission occurred in $27.3 \%$ at 12 months and, in $14.3 \%$, at 24 months. Male gender presented a lower rate of total remission compared with women at 12 months (45.5\% versus $81.6 \%, p=0.007$ ). Among several factors studied, none was correlated with total or partial remission at 12 months. The subgroup "clinical class IV" presented a higher frequency of total remission than the subgroup "histological class IV". Conclusion: Our therapeutic approach achieved an excellent outcome in two years. Correlations between clinical and laboratory variables and total or partial remission were not observed. Male gender presented a lower rate of total remission compared with women. Despite the small number of patients studied and the controversies of renal biopsy, the rate of total remission was higher in patients with "clinical class $I V$ " than in those with "histological class IV".

Keywords: systemic lupus erythematosus, nephritis, complete remission, renal biopsy.

Este trabalho foi desenvolvido para demonstrar a experiência de um serviço terciário no tratamento da indução e manutenção da remissão da nefrite lúpica. Sabe-se que esse é um dos temas de maior relevância na Reumatologia, apesar do pequeno número de publicações sobre o assunto em nosso meio, somado às controvérsias quanto à indicação de biópsia renal nesses pacientes. Os objetivos principais deste estudo são determinar a frequência de remissão total e parcial da nefrite aos 12 e 24 meses de acompanhamento; comparar esses subgrupos aos 12 meses, correlacionando as variáveis renais iniciais com a resposta terapêutica e demonstrando a experiência de um serviço brasileiro no manejo das manifestações renais do LES. Outro objetivo é comparar os resultados terapêuticos dos subgrupos de pacientes com glomerulonefrite proliferativa difusa por correlação clínico-patológica ("classe IV clínica") daqueles com "classe IV histológica", ou seja, com biópsia renal.

\section{MATERIAL E MÉTODOS}

Trata-se de uma análise retrospectiva de prontuários de pacientes com diagnóstico de LES e nefrite, segundo os critérios de classificação do ACR de 1997, atendidos no Serviço de Reumatologia da Santa Casa de Misericórdia de São Paulo, no período de 1990 a 2003. A nefrite lúpica é definida pela presença de proteinúria persistente ( $>0,5 \mathrm{~g}$ nas 24 horas) ou maior que $3+$ no EAS ou pela cilindrúria (cilindros hemáticos, tubulares, granulosos ou mistos). 
Tabela 1

Correlação entre achados clínico-laboratoriais e classificação histológica (OMS) da nefrite lúpica

\begin{tabular}{lllll}
\hline & Classe II & Classe III & Classe IV & Classe V \\
\hline Hipertensão arterial & Ausente & Rara & Frequente & Rara \\
Proteinúria (gramas/24horas) & $<1$ & $<2$ & $1-20$ & $3,5-20$ \\
$\begin{array}{l}\text { Hematúria } \\
\text { (hemácias/por campo) }\end{array}$ & $5-15$ & $5-15$ & $>15$ & Ausente \\
Leucocitúria (leucócitos por campo) & $5-15$ & $5-15$ & $>15$ & Ausente \\
Creatinina (mg/dL) & Normal & $\begin{array}{l}\text { Normal ou } \\
\text { aumentada }\end{array}$ & $\begin{array}{l}\text { Frequentemente } \\
\text { aumentada }\end{array}$ & Normal \\
Taxa de Filtração Glomerular (ml/min) & Normal & $60-80$ & $<60$ & Normal \\
CH50 & $\downarrow$ & $\downarrow$ & $\downarrow \downarrow$ & Normal \\
C3 & $\downarrow$ & $\downarrow$ & $\downarrow \downarrow$ & Normal \\
Anti-dsDNA & $\uparrow$ & $\uparrow$ & $\uparrow \uparrow$ & Normal \\
\hline
\end{tabular}

Adaptado de Esdaile, 1991 e 1992

Foram incluídos os pacientes que realizaram ou não biópsia renal inicial. A classificação de nefrite foi estabelecida clinicamente de acordo com Esdaile ${ }^{4}$ ou por histopatologia. A indicação da biópsia renal foi individualizada e se baseou nas seguintes condições: dificuldade em se estabelecer uma correlação clínico-patológica (casos duvidosos), presença de síndrome nefrótica e/ou para auxiliar na decisão terapêutica.

Os pacientes foram acompanhados por dois anos a partir do diagnóstico de nefrite e do início do tratamento, que se baseou na correlação clínico-patológica ou nos dados da biópsia renal. O esquema terapêutico do nosso serviço utilizou a prednisona em altas doses (prednisona $1-2 \mathrm{mg} / \mathrm{kg} /$ dia por via oral) em todos os pacientes ou a metilprednisolona, na dose de $1 \mathrm{~g} / \mathrm{dia}$ por via intravenosa, durante 3 a 5 dias consecutivos, em casos mais graves. A ciclofosfamida intravenosa (IV) foi utilizada na dose de 0,5 a $1 \mathrm{~g} / \mathrm{m}^{2}$ de superfície corporal como o imunossupressor de primeira escolha para a indução de remissão em todos os pacientes com classe III, IV ou V. Outros agentes como azatioprina ou micofenolato mofetil (MMF) foram usados para o tratamento de manutenção da nefrite após 6-9 meses de ciclofosfamida IV mensal ou em casos de falha ou intolerância ao esquema anteriormente descrito. Os pacientes portadores de glomerulonefrite mesangial foram tratados apenas com corticosteroide em altas doses.

Foi avaliada a resposta terapêutica após 12 e 24 meses. Os critérios de Boumpas e Balow (1998) foram utilizados para a classificação da resposta terapêutica como total, parcial ou sem resposta. ${ }^{5}$ Segundo esses autores, a remissão total é definida como a estabilização ou normalização da creatinina sérica, sedimento urinário com número inferior a cinco hemácias por campo e proteinúria de 24 horas menor que $1,0 \mathrm{~g}$ por seis meses, no mínimo. A remissão parcial caracteriza-se por estabilização ou melhora da creatinina sérica, hematúria menor que cinco células por campo e redução persistente da proteinúria de 24 horas (se nefrótica, redução maior ou igual a 50\%, mas com valor menor que 3,0 g/24 horas; se não-nefrótica, redução maior ou igual a $50 \%$, mas com valor maior que $1,0 \mathrm{~g} / 24$ horas). Quando ocorre deterioração da função renal (excluídas outras causas como sepse, drogas nefrotóxicas, trombose de veia renal), aumento ou diminuição da proteinúria que não preenche os critérios acima, considera-se como sem resposta.

Os portadores de comorbidades que comprometem os rins foram excluídos do estudo. Foram analisadas as variáveis demográficas, clínicas, laboratoriais e o índice de atividade da doença (SLEDAI). Para verificar a influência de diferentes fatores no prognóstico da nefrite, os pacientes foram distribuídos em grupos de remissão total ou parcial, "classe IV clínica" e "classe IV histológica".

Os dados foram expressos como média \pm desvio padrão. O programa utilizado para a análise estatística foi o SPSS versão 13.0. A comparação entre médias foi realizada utilizando-se o teste $t$ de Student e a comparação entre frequências foi feita com o teste qui-quadrado e Mc Nemar. Considerou-se a diferença estatisticamente significante quando $p<0,05$.

Para a realização do presente estudo, foi obtida a aprovação prévia do Comitê de Ética em Pesquisa da instituição.

\section{RESULTADOS}

Foram estudados os registros de 100 pacientes com nefrite lúpica durante dois anos. A idade média dos pacientes na época do diagnóstico de LES foi de 24,71 $\pm 10,14$ anos, variando de 
5 a 46 anos. A idade média ao diagnóstico de nefrite lúpica foi de $26,78 \pm 10,95$ anos, com uma variação de 5 a 53 anos. $O$ intervalo de tempo entre o diagnóstico de LES e o de nefrite foi de 2,32 $\pm 4,70$ anos. Nessa casuística, $88 \%$ eram mulheres e houve predomínio da raça branca (Tabela 2).

As principais manifestações extrarrenais do LES foram articulares, cutâneo-mucosas, hematológicas e serosite, encontradas em $81 \%, 79 \%, 67 \%$ e $24 \%$ dos casos, respectivamente. Lúpus neuropsiquiátrico ocorreu em $11 \%$ dos doentes. $\mathrm{O}$ valor médio do SLEDAI ao diagnóstico foi de 16,09 $\pm 6,48$, expressando doença em considerável atividade, isto é, SLE$\mathrm{DAI} \geq 4$.

Em relação ao quadro renal inicial, o valor médio da creatinina sérica foi de $1,02 \pm 0,49 \mathrm{mg} / \mathrm{dL}$, variando de $0,4-3,6 \mathrm{mg} / \mathrm{dL}$ e a proteinúria média foi de $2,57 \pm 2,39$ gramas/24 horas. Ao diagnóstico de nefrite, $33 \%$ dos pacientes eram hipertensos de acordo com os critérios propostos pela $\mathrm{OMS}^{6}$ e $66 \%$ tinham o anticorpo anti-dsDNA presente em altos títulos (Tabela 3 ).

Cinquenta e seis pacientes realizaram biópsia renal percutânea. Os subtipos histológicos mais frequentes foram classe II e IV (33,9\% e 32,2\%, respectivamente). A glomerulonefrite classe III foi observada em 11 dos 56 pacientes (19,6\%) e apenas oito pacientes apresentavam classe V (14,3\%). Dos 44 pacientes que não foram submetidos à biópsia renal, seis tinham dados clínico-laboratoriais compatíveis com glomerulonefrite mesangial, $11 \mathrm{com}$ glomerulonefrite proliferativa-focal, $23 \mathrm{com}$ proliferativa difusa e 4 com membranosa.

No que se refere ao tratamento, todos os pacientes receberam corticosteroide para a nefrite, sendo a prednisona utilizada em $100 \%$ dos casos. Do total de 100 pacientes, $75 \%$ utilizaram a ciclofosfamida em pulsos intravenosos, pois apresentavam glomerulonefrite classe III, IV ou V por correlação clínicohistológica, ou por biópsia renal, com dose cumulativa de $9,44 \pm 4,22$ gramas. Quinze pacientes receberam pulsoterapia com ciclofosfamida e metilprednisolona. Dentre os pacientes que utilizaram a ciclofosfamida, $60(80 \%)$ completaram o período de indução da remissão e, posteriormente, receberam a azatioprina na dose de $2-3 \mathrm{mg} / \mathrm{kg} /$ dia para o tratamento de manutenção. Quinze pacientes descontinuaram a ciclofosfamida por intolerância ou efeitos adversos, sendo os principais gastrintestinais (náuseas e vômitos em oito pacientes), infecções (cinco pacientes) e leucopenia grave $\left(<2000\right.$ células $/ \mathrm{mm}^{3}$, em quatro indivíduos). Nos casos de interrupção da ciclofosfamida durante a fase de indução, a azatioprina foi administrada em 10 pacientes, e o MMF foi usado em cinco casos, na dose de $2 \mathrm{~g}$ /dia, divididos em duas tomadas. A maioria dos pacientes com manifestações cutâneo-articulares (85\%) fez uso de

\section{Tabela 2}

Características dos 100 pacientes com nefrite lúpica acompanhados durante 2 anos

\begin{tabular}{lc}
\hline $\begin{array}{l}\text { Idade ao diagnóstico de LES em anos } \\
(\mathrm{M} \pm \mathrm{DP})\end{array}$ & $24,71 \pm 10,14$ \\
$\begin{array}{l}(5-46) \\
\text { Idade ao diagnóstico de nefrite em anos } \\
(\mathrm{M} \pm \mathrm{DP})\end{array}$ & $26,78 \pm 10,95$ \\
$\begin{array}{l}\text { Intervalo entre o diagnóstico de LES e } \\
\text { o diagnóstico de nefrite em anos }\end{array}$ & $2,32 \pm 4,70$ \\
$(\mathrm{M} \pm \mathrm{DP})$ & $(0-24)$ \\
Sexo feminino (\%) & \\
Raça (\%) & $88(88 \%)$ \\
Branca & \\
Outras & $57(57 \%)$ \\
Não registrada & $29(29 \%)$ \\
\hline
\end{tabular}

LES = Lúpus eritematoso sistêmico; $M$ = Média; DP = Desvio-padrão.

\section{Tabela 3}

Dados clínicos e laboratoriais iniciais de 100 pacientes com nefrite lúpica

\begin{tabular}{lc}
\hline Creatinina em mg/dL & $1,02 \pm 0,49$ \\
$(\mathrm{M} \pm$ DP/variação) & $(0,4-3,6)$ \\
Proteinúria em g/24h & $2,57 \pm 2,39$ \\
$(\mathrm{M} \pm \mathrm{DP} /$ variação) & $(0-10,3)$ \\
HAS (\%) & $37(37 \%)$ \\
Positividade do Anti-dsDNA (\%) & $66(66 \%)$ \\
\hline
\end{tabular}

M = média; DP = desvio-padrão; HA S= Hipertensão Arterial Sistêmica; Anti-dsDNA = anti DNA de dupla hélice.

antimaláricos (hidroxicloroquina $6 \mathrm{mg} / \mathrm{kg} /$ dia ou difosfato de cloroquina $4 \mathrm{mg} / \mathrm{kg} / \mathrm{dia})$.

As variáveis renais analisadas foram: creatinina sérica, proteinúria de 24 horas, alterações no sedimento urinário e frações do complemento. Comparando-se os resultados dessas variáveis ao início e após 12 meses de acompanhamento, verificou-se que todos os pacientes apresentaram redução significativa da proteínúria de 24 horas, melhora do sedimento urinário e aumento nos valores das frações do complemento (Tabela 4). Durante o acompanhamento, houve tendência para o aumento da creatinina sérica, sem, no entanto, atingir significância estatística.

A frequência de remissão total aos 12 meses foi $72,7 \%$ e aos 24 meses, $85,7 \%(p=0,013)$. A remissão parcial foi atingida em $27,3 \%$ dos pacientes aos 12 meses e em $14,3 \%$ aos 24 meses (Figura 1). A comparação entre os subgrupos com remissão total e parcial aos 12 meses e as variáveis do LES e da nefrite ao início demonstrou que nenhuma variável se correlacionou com a resposta ao tratamento (Tabela 5). Houve exacerbação renal durante o tratamento da nefrite em $40 \%$ dos casos, sendo 
a maioria (80\%) durante a fase de manutenção. Nesses casos, as doses do glicorticoide e do imunossupressor foram otimizadas, respeitando o intervalo de doses descrito anteriormente.

O sexo masculino apresentou menores taxas de remissão total aos 12 meses comparado ao feminino (45,5\% versus $81,6 \%$, respectivamente, $p=0,007)$, o que poderia sugerir uma evolução menos favorável da nefrite lúpica entre os homens dessa população (Figura 2). Como se pode constatar pelos dados expostos, os homens que não atingiram remissão total aos 12 meses, evoluíram com resposta parcial ao tratamento instituído nesse período.

\section{Tabela 4}

Comparação das variáveis relacionadas à nefrite ao início $\left(\mathrm{T}_{0}\right)$ e após 12 meses $\left(\mathrm{T}_{12}\right)$ em 100 pacientes com nefrite lúpica

\begin{tabular}{|c|c|c|c|}
\hline & $\mathrm{T}_{0}$ & $\mathrm{~T}_{12}$ & $P^{*}$ \\
\hline $\begin{array}{l}\text { Creatinina em mg/dL } \\
(M \pm D P)\end{array}$ & $0,99 \pm 0,42$ & $1,03 \pm 0,94$ & 0,663 \\
\hline $\begin{array}{l}\text { Proteinúria em g/24h } \\
(\mathrm{M} \pm \mathrm{DP})\end{array}$ & $2,58 \pm 2,42$ & $1,26 \pm 1,83$ & 0,000 \\
\hline $\begin{array}{l}\text { Hematúria em cel } p / c \\
(M \pm D P)\end{array}$ & $22,99 \pm 23,42$ & $13,06 \pm 18,64$ & 0,000 \\
\hline $\begin{array}{l}\text { Leucocitúria em cel p/c } \\
(M \pm D P)\end{array}$ & $14,61 \pm 16,77$ & $10,10 \pm 15,34$ & 0,037 \\
\hline $\mathrm{CH} 50(\mathrm{M} \pm \mathrm{DP})$ & $\begin{array}{c}172,50 \pm \\
73,42\end{array}$ & $\begin{array}{c}190,67 \pm \\
89,83\end{array}$ & 0,031 \\
\hline$C 4(M \pm D P)$ & $16,96 \pm 8,04$ & $21,48 \pm 9,86$ & 0,024 \\
\hline $\mathrm{C} 3(\mathrm{M} \pm \mathrm{DP})$ & $82,44 \pm 39,36$ & $\begin{array}{c}100,12 \pm \\
33,49\end{array}$ & 0,026 \\
\hline
\end{tabular}

* Teste $t$ de student

$\mathrm{M}=$ média; DP = desvio-padrão; cel p/c = células por campo;

$\mathrm{CH} 50$ = complemento hemolítico total sérico; C4 = complemento 4; C3 = complemento 3.

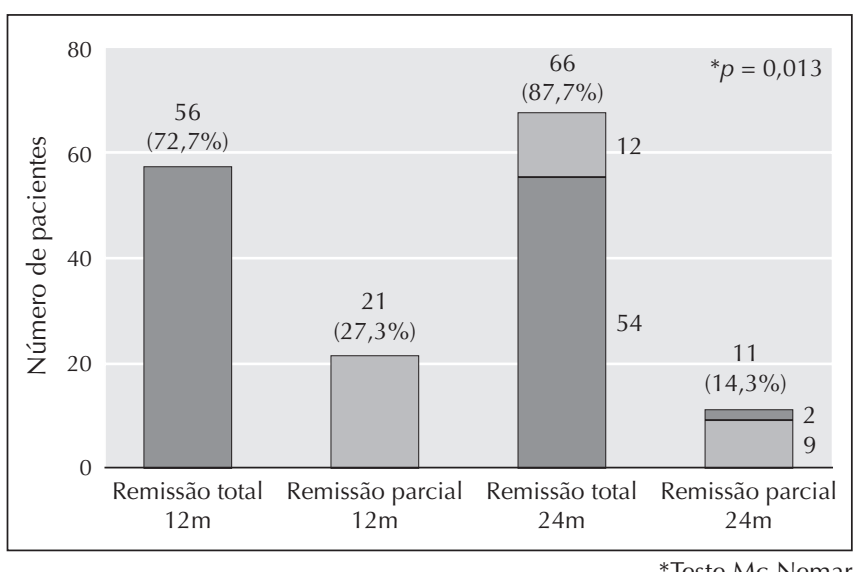

*Teste Mc Nemar

Figura 1. Comparação entre os subgrupos com remissão total e parcial aos $12 \mathrm{e}$ 24 meses de acompanhamento $(\mathrm{n}=77)$.
A análise comparativa entre os grupos com "classe IV clínica" e "classe IV histológica" e as variáveis iniciais do LES e da nefrite evidenciou que não há diferença estatística entre os valores de SLEDAI, creatinina sérica e proteinúria de 24 horas, bem como em relação ao sedimento urinário, aos valores das frações do complemento e à dose cumulativa da ciclofosfamida nos dois grupos (Tabela 6). Foi observada uma frequência significativamente maior de remissão total nos pacientes com "classe IV clínica" em relação àqueles com "classe IV histológica" (Figura 3).

\section{DISCUSSÃO}

Neste estudo, determinou-se a evolução de 100 pacientes com nefrite lúpica acompanhados por dois anos. O esquema terapêutico do nosso serviço utilizou a ciclofosfamida IV como o imunossupressor de primeira escolha para a indução de remissão da nefrite classe III, IV ou V. Outros agentes como azatioprina ou MMF foram usados para o tratamento de manutenção da nefrite após 6-9 pulsos de ciclofosfamida IV mensal ou em casos de falha ou intolerância ao esquema anteriormente

\section{Tabela 5}

Comparação entre os subgrupos com remissão total e parcial aos 12 meses e as variáveis do LES e da nefrite no início do acompanhamento: fatores prognósticos

\begin{tabular}{|c|c|c|c|}
\hline & $\begin{array}{l}\text { Remissão Total } \\
\quad(n=76)\end{array}$ & $\begin{array}{l}\text { Remissão Parcial } \\
\quad(n=22)\end{array}$ & $* p$ \\
\hline Idade em anos $(M \pm D P)$ & $26,57 \pm 11,33$ & $27,90 \pm 9,04$ & 0,570 \\
\hline $\operatorname{SLEDAI}(M \pm D P)$ & $16,17 \pm 6,57$ & $15,77 \pm 6,47$ & 0,802 \\
\hline $\begin{array}{l}\text { Creatinina em mg/ } \\
d L(M \pm D P)\end{array}$ & $0,94 \pm 0,86$ & $1,13 \pm 0,55$ & 0,147 \\
\hline $\begin{array}{l}\text { Proteinúria em g/24h } \\
(M \pm D P)\end{array}$ & $2,31 \pm 2,19$ & $3,49 \pm 2,94$ & 0,093 \\
\hline $\begin{array}{l}\text { Hematúria em cel p/c } \\
(M \pm D P)\end{array}$ & $21,61 \pm 22,38$ & $27,77 \pm 26,73$ & 0,332 \\
\hline $\begin{array}{l}\text { Leucocitúria em cel p/c } \\
(M \pm D P)\end{array}$ & $12,20 \pm 13,35$ & $22,95 \pm 23,87$ & 0,064 \\
\hline $\mathrm{CH} 50(\mathrm{M} \pm \mathrm{DP})$ & $163,97 \pm 119,62$ & $140,75 \pm 111,77$ & 0,614 \\
\hline $\mathrm{C} 3(\mathrm{M} \pm \mathrm{DP})$ & $78,39 \pm 34,61$ & $84,15 \pm 42,68$ & 0,663 \\
\hline $\mathrm{C} 4(\mathrm{M} \pm \mathrm{DP})$ & $16,28 \pm 8,63$ & $16,08 \pm 8,01$ & 0,938 \\
\hline $\begin{array}{l}\text { Dose cumulativa } \\
\text { de ciclofosfamida } \\
\text { em } g(M \pm D P)\end{array}$ & $9,33 \pm 3,84$ & $10,27 \pm 4,77$ & 0,42 \\
\hline \multicolumn{4}{|l|}{ * Teste $t$ de student } \\
\hline \multicolumn{4}{|c|}{$\begin{array}{l}\text { LES = Lúpus eritematoso sistêmico; } M=\text { média; DP = desvio-padrão; } \\
\text { SLEDAl = Systemic Lupus Erythematosus Disease Activity Index; } \\
\text { Cel p/c = células por campo; CH50 = complemento hemolítico total sérico; } \\
\text { C4 = complemento 4; C3 = complemento } 3 .\end{array}$} \\
\hline
\end{tabular}




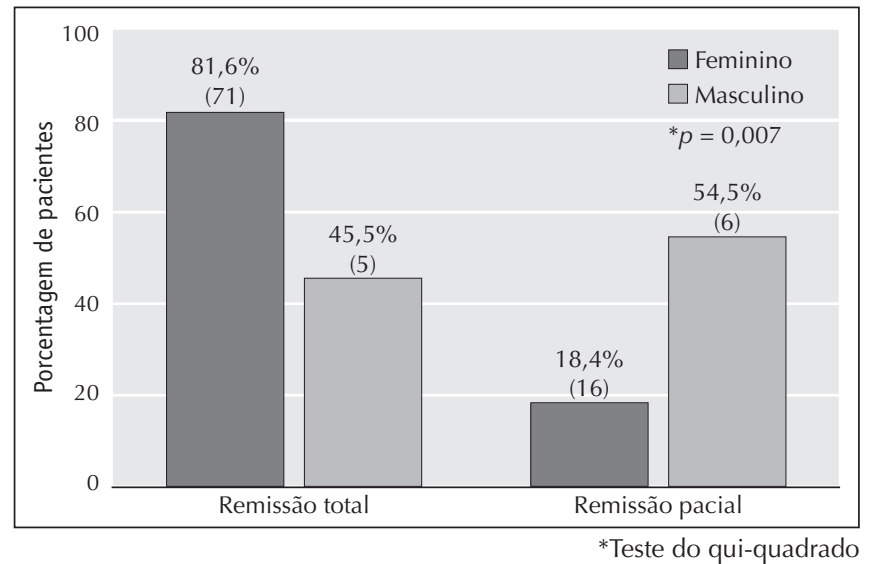

Figura 2. Comparação entre os subgrupos do sexo feminino e masculino quanto à remissão total e parcial aos 12 meses.

\section{Tabela 6}

\begin{tabular}{|c|c|c|c|}
\hline & \multicolumn{2}{|c|}{ Classe IV } & \multirow[b]{2}{*}{${ }^{*} p$} \\
\hline & $\begin{array}{l}\text { Histológica } \\
(\mathrm{n}=18)\end{array}$ & $\begin{array}{l}\text { Clínica } \\
(\mathrm{n}=23)\end{array}$ & \\
\hline $\operatorname{SLEDAI}(M \pm D P)$ & $16,22 \pm 5,26$ & $17,83 \pm 5,67$ & 0,356 \\
\hline $\begin{array}{l}\text { Creatinina em } \\
\mathrm{mg} / \mathrm{dL}(\mathrm{M} \pm \mathrm{DP})\end{array}$ & $1,13 \pm 0,33$ & $1,09 \pm 0,37$ & 0,707 \\
\hline $\begin{array}{l}\text { Proteinúria em g/24h } \\
(M \pm D P)\end{array}$ & $2,67 \pm 2,12$ & $3,37 \pm 2,70$ & 0,360 \\
\hline $\begin{array}{l}\text { Hematúria em cel p/c } \\
(M \pm D P)\end{array}$ & $37,33 \pm 25,22$ & $34,96 \pm 26,33$ & 0,771 \\
\hline $\begin{array}{l}\text { Leucocitúria em cel p/c } \\
(M \pm D P)\end{array}$ & $15,61 \pm 12,77$ & $19,78 \pm 23,44$ & 0,472 \\
\hline $\mathrm{CH} 50(\mathrm{M} \pm \mathrm{DP})$ & $135,71 \pm 119,62$ & $124,22 \pm 83,65$ & 0,833 \\
\hline $\mathrm{C} 3(\mathrm{M} \pm \mathrm{DP})$ & $86,76 \pm 46,82$ & $58,53 \pm 19,17$ & 0,117 \\
\hline $\mathrm{C} 4(\mathrm{M} \pm \mathrm{DP})$ & $15,00 \pm 6,61$ & $13,07 \pm 6,90$ & 0,511 \\
\hline $\begin{array}{l}\text { Dose cumulativa } \\
\text { de ciclofosfamida } \\
\text { em g }(M \pm D P)\end{array}$ & $8,72 \pm 3,79$ & $10,31 \pm 4,48$ & 0,227 \\
\hline
\end{tabular}

$\mathrm{M}=$ Média; DP = Desvio-padrão; SLEDAl = Systemic Lupus Erythematosus Disease Activity Index; Cel p/c = Células por campo; $\mathrm{CH} 50=$ Complemento hemolítico total sérico; $\mathrm{C} 4$ = complemento $4 ; \mathrm{C} 3=$ complemento 3 .

descrito. Segundo Contreras et al., ${ }^{6}$ os pacientes com nefrite lúpica proliferativa difusa que recebem ciclofosfamida IV por curto período seguida por terapia de manutenção com azatioprina ou MMF apresentam resultados mais eficazes e maior segurança que o uso prolongado de ciclofosfamida. Houssiau corroborou que a ciclofosfamida IV é a única terapia capaz de reduzir a evolução para doença renal terminal nesses pacientes em estudos com longo período de acompanhamento. ${ }^{7}$

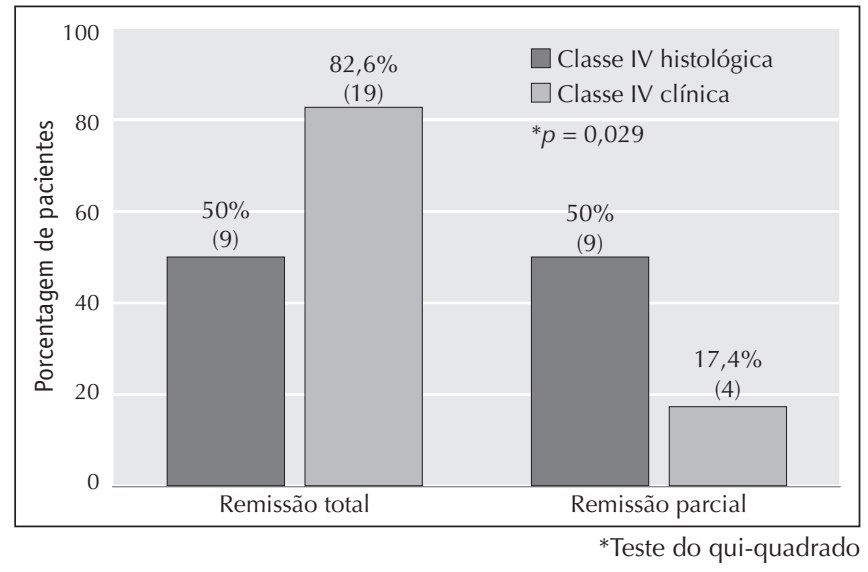

Figura 3. Comparação entre os subgrupos com "classe IV clínica" e "classe IV histológica" quanto à remissão total e parcial aos 12 meses de acompanhamento.

Na nossa casuística, todos os pacientes demonstraram evolução satisfatória ao final de 12 meses de acompanhamento. Dentre as variáveis renais analisadas, houve redução significativa da proteinúria de 24 horas, melhora do sedimento urinário e aumento dos valores do complemento (C3, $\mathrm{C} 4$ e CH50). A frequência de remissão total aos 12 meses foi $72,7 \%$ e aos 24 meses, $85,7 \%(p=0,013)$. Segundo Urowitz et al., os pacientes com nefrite lúpica em atividade tratados com ciclofosfamida ou outro imunossupressor apresentam remissão total em aproximadamente $70 \%$ dos casos. ${ }^{8}$

De acordo com os dados expostos, nenhum paciente apresentou critérios clínico-laboratoriais de ausência de resposta à terapêutica ao final de dois anos de acompanhamento. No entanto, esse dado deve ser interpretado com cautela, uma vez que, durante o período de estudo, houve recorrência de doença renal ativa em aproximadamente $40 \%$ dos casos, contudo, tais pacientes evoluíram satisfatoriamente com a otimização do tratamento (aumento da dose do corticosteroide e/ou do imunossupressor).

A comparação entre os subgrupos com remissão total e parcial aos 12 meses e as variáveis iniciais do LES e da nefrite constatou que nenhum dado se correlacionou com a resposta ao tratamento. Estudos anteriores demonstraram que variáveis como idade, proteinúria, hematúria e presença de hipertensão arterial na época do diagnóstico têm valor prognóstico questionável na evolução da nefrite lúpica., ${ }^{9,11,11,12}$ Outros autores concluíram em seus estudos que creatinina elevada e hipoalbuminemia representam fatores preditores de pior prognóstico renal. ${ }^{13,14} \mathrm{Em}$ trabalho publicado no Brasil, ${ }^{15}$ verificou-se que, em portadores de LES de início após os 16 anos, piores desfechos ocorreram naqueles com nefrite, manifestada clinicamente no início ou na evolução da doença, 
com hipertensão arterial no início da doença, com o anti-DNA positivo e com o uso de ciclofosfamida. Possíveis explicações para esses fatos discrepantes decorrem, principalmente, de diferenças metodológicas das séries: critérios de inclusão e seleção de pacientes, estudos retrospectivos, tempos de acompanhamento, tratamentos realizados, definição dos desfechos clínicos, tamanho da amostra, entre outros.

O sexo masculino apresentou menores taxas de remissão total aos 12 meses comparado ao feminino, o que poderia sugerir pior prognóstico da nefrite lúpica entre os homens na nossa casuística. Esse dado difere da literatura em que se demonstra que o sexo do indivíduo não apresenta valor prognóstico na evolução da nefrite lúpica. ${ }^{16}$

Apesar do pequeno número de pacientes estudados e das controvérsias quanto à biópsia renal inicial, as taxas de remissão nos pacientes com "classe IV clínica" e "classe IV histológica" foram altas aos 12 meses. Um balanço entre os ganhos potenciais de informações que podem guiar um esquema terapêutico e as complicações inerentes ao procedimento invasivo sempre deve ser levado em consideração antes de indicar a biópsia renal. Estudos prévios demonstraram que parâmetros clínico-laboratoriais são capazes de predizer com acurácia o prognóstico da nefrite lúpica, sem a necessidade de biópsia em todos os casos com envolvimento renal. ${ }^{1718}$ Já outros estudos advogam que os dados histológicos oferecem informações adicionais aos parâmetros laboratoriais e podem guiar, de maneira mais precisa, a escolha terapêutica. ${ }^{19} \mathrm{Como}$ pode ser percebido, o tema permanece controverso. Na experiência do nosso serviço, a biópsia renal é individualizada e indicada, sobretudo, nas seguintes situações: dúvida quanto à correlação clínico-histológica, presença de síndrome nefrótica, ou em casos que não responderam à terapia de indução com ciclofosfamida. Pelo presente estudo, a frequência de remissão total aos 12 meses foi significativamente mais alta nos pacientes com "classe IV clínica", sem a necessidade de informações adicionais pelo exame histológico. Na casuística apresentada, as variáveis renais e do LES, bem como a dose cumulativa da ciclofosfamida não diferiram entre os pacientes com "classe IV histológica" ou "clínica", isto é, com e sem biópsia renal.

Portanto, concluímos que, neste estudo, a realização de biópsia renal para a determinação do subtipo histológico não influenciou na resposta ao tratamento em pacientes com nefrite lúpica acompanhados por dois anos. Os dados apresentados demonstraram que a correlação clínico-histológica pode ajudar na decisão terapêutica desses pacientes, reservando a biópsia renal apenas para situações especiais.

\section{Analysis of 100 patients with lupus nephritis followed up for 2 years}

\section{INTRODUCTION}

Systemic lupus erythematosus (SLE) is an autoimmune chronic inflammatory disease that may affect multiple systems. Renal involvement in SLE is a significant cause of morbidity and mortality due to the possibility of progression to end stage renal disease (ESRD) and treatment-related complications. By the criteria of the American College of Rheumatology (ACR - 1997), lupus nephritis is defined by the presence of persistent proteinuria ( $>0,5 \mathrm{~g}$ in 24 hours), or higher than $3+$, or by cylindruria (hematic, tubular, granular or mix cylinder). Among histological standards created by World Health Organization (WHO), diffuse proliferative glomerulonephritis (class IV) represents the most prevalent and worst prognostic subtype. ${ }^{1}$ Nephritis is the main cause of hospitalization and mortality among SLE patients. ${ }^{2}$

Controlled trials have shown that the use of cyclophosphamide for lupus nephritis treatment is correlated with higher rates of long term renal function preservation and lower incidence of ESRD. ${ }^{3}$

There are controversies about indicating renal biopsy to every lupus patients with nephritis. Esdaile et al. established a correlation between clinical and laboratory findings and histological classification of lupus nephritis, in order to guide therapeutic approach and rationalize the performance of invasive procedures in such population. ${ }^{4}$ (Table 1)

This study was designed to demonstrate a tertiary hospital experience in the treatment of induction and maintenance of remission of lupus nephritis. This is one of the most relevant topics in Rheumatology, although there are few publications on it, added to the controversy about the indication of renal biopsy in these patients. The main objectives of this study are to establish the frequency of complete and partial remission of nephritis both at 12 and 24 months; to compare these subgroups at 12 months, with correlation between initial renal variables with therapeutic response; and to demonstrate the experience of a Brazilian centre in treating SLE renal manifestations. Another endpoint is to compare the therapeutic results of the subgroup with diffuse proliferative glomerulonephritis by clinicopathological correlation ("clinical class IV") with those with biopsy-proven WHO class IV ("histological class IV"). 
Table 1

Correlation between clinical and laboratory findings and histological classification of lupus nephritis

\begin{tabular}{lllll}
\hline & Class II & Class III & Class IV & Class V \\
\hline Arterial hypertension & None & Rare & Frequent & Rare \\
Proteinuria (grams/24hours) & $<1$ & $<2$ & $1-20$ & $3,5-20$ \\
$\begin{array}{l}\text { Hematuria } \\
\text { Erythrocytes/per field) }\end{array}$ & $5-15$ & $5-15$ & $>15$ & None \\
Leucocyturia/leucocytes per field) & $5-15$ & $5-15$ & $>15$ & None \\
Creatinine (mg/dl) & Normal & $\begin{array}{l}\text { Normal or } \\
\text { incremented }\end{array}$ & $\begin{array}{l}\text { Frequently } \\
\text { incremented }\end{array}$ & Normal \\
Glomerular filtration rate (ml/min) & Normal & $60-80$ & $<60$ & Normal \\
CH50 & $\downarrow$ & $\downarrow$ & $\downarrow \downarrow$ & Normal \\
C3 & $\downarrow$ & $\downarrow$ & $\downarrow \downarrow$ & Normal \\
Anti-dsdna & $\uparrow$ & $\uparrow$ & $\uparrow \uparrow$ & Normal \\
\hline
\end{tabular}

Esdaile, 1991 and 1992

\section{MATERIAL AND METHODS}

Retrospective chart analysis was done for patients with SLE and nephritis who attended the Rheumatology Department at Santa Casa de Misericórdia de São Paulo from 1990 to 2003. Lupus nephritis is defined according the ACR criteria as persistent proteinuria ( $>0,5 \mathrm{~g}$ in 24 hours) or higher than $3+$ or by cylindruria (hematic, tubular, granular or mix cylinders).

Patients who had been submitted to initial renal biopsy or not have been included. Classification of nephritis had been clinically established according to Esdaile ${ }^{4}$ or by histopathology. Renal biopsy was individually assigned based, mainly, on the following situations: difficulty to establish a clinicopathological correlation (doubtful cases), presence of nephrotic syndrome and/or to support therapeutic decision.

Patients had been followed up for two years since nephritis was diagnosed and therapy started, based on clinicopathological correlation or on renal biopsy data. Our therapeutic approach included high doses of corticosteroids (prednisone $1-2 \mathrm{mg} / \mathrm{kg}$ /day orally) in every patient or $1 \mathrm{~g} /$ day doses of methylprednisolone intravenously, during 3-5 consecutive days, in more severe cases. Intravenous (IV) monthly cyclophosphamide was used in 0.5 to $1 \mathrm{~g} / \mathrm{m}^{2}$ doses of body surface area as a first-choice of immunosuppressive agent for induction of class III, IV or V nephritis remission. Azathioprine or mycophenolate mofetil (MMF) were used for nephritis maintenance treatment after 6-9 months of cyclophosphamide IV, or in case of failure or intolerance to the approach described before. Patients with mesangial glomerulonephritis were treated only with high doses of glucocorticoid.

Therapeutic response was evaluated after 12 and 24 months. Boumpas \& Balow (1998) criteria were used to classify therapeutic response as complete, partial and no response. ${ }^{5}$ According to these authors, complete remission is defined as stabilization or normalization of serum creatinine, urinary sediment with less than five erythrocytes per field and 24-hour proteinuria lower than $1.0 \mathrm{~g}$ for, at least, six months. Partial remission is characterized by stabilization or improvement of serum creatinine, hematuria lower than five cells per field and persistent 24-hour proteinuria reduction (if nephrotic, reduction higher than or equal to $50 \%$, but with value lower than $3,0 \mathrm{~g} / 24$ hours; if non nephrotic, reduction higher than or equal to $50 \%$, but with value higher than $1.0 \mathrm{~g} / 24$ hours). When presenting with renal function deterioration (excluding other causes, like sepsis, nephrotoxic drugs, renal vein thrombosis), proteinuria increment or reduction not meeting criteria above, patients were considered as no response.

Patients with comorbidities that compromise the kidneys were excluded from this study. The demographic, clinical and laboratory variables and the Systemic Lupus Erythematosus Disease Activity Index (SLEDAI) were analyzed. In order to verify the influence of different factors in nephritis prognosis, patients were divided into groups of complete and partial remission, "clinical class IV" and "histological class IV".

Data were expressed as mean \pm standard deviation. The program used for statistical analysis was SPSS version 13.0. Comparison between means was performed using " $t$ " Student test, and comparison between frequencies was done with McNemar and chi-square tests. When $p<0,05$, difference was considered significant.

This study had been previously approved by the Research Ethics Committee of the institution. 


\section{RESULTS}

Records of 100 patients with lupus nephritis were analyzed during two years. The average age at SLE diagnosis was 24.71 \pm 10.14 years, ranging from 5 to 46 years old. The average age at lupus nephritis diagnosis was $26.78 \pm 10.95$ years, ranging from 5 to 53 years. Period of time between SLE and nephritis diagnosis was $2.32 \pm 4.70$ years. In this trial, $88 \%$ were female and predominantly caucasian (Table 2 ).

The main extra-renal SLE manifestations were joints, mucous-cutaneous, hematologic and serositis, found in $81 \%$, $79 \%, 67 \%$ and $24 \%$ of the patients. Neuropsychiatric lupus occurred in $11 \%$ of patients. The average SLEDAI value at diagnosis was $16.09 \pm 6.48$, expressing disease in considerable activity, that is, SLEDAI $\geq 4$.

Baseline average serum creatinine was $1.02 \pm 0.49 \mathrm{mg} / \mathrm{dL}$, ranging from 0.4 to $3.6 \mathrm{mg} / \mathrm{dL}$, and average proteinuria was $2.57+2.39$ grams $/ 24$ hours. At the time of nephritis diagnosis, $33 \%$ of patients were hypertensive according to criteria proposed by World Health Organization ${ }^{6}$ and $66 \%$ had high titers of antibody anti-dsDNA (Table 3).

Fifty-six patients had been submitted to percutaneous renal biopsy. The most frequent histological subtypes were WHO class II and IV (33.9\% and 32.2\%, respectively). Glomerulonephritis class III was observed in 11 of 56 patients (19.6\%) and only eight patients presented class V (14.3\%). Out of 44 patients who had not been submitted to renal biopsy, 6 had clinical and laboratory data compatible to mesangial glomerulonephritis, 11 with focal proliferative glomerulonephritis, 23 with diffuse proliferative and 4 with membranous.

Regarding the treatment, all patients received glucocorticoid for nephritis, and prednisone had been used in 100\% of the cases. Out of 100 patients, $75 \%$ used IV cyclophosphamide, since they presented glomerulonephritis class III, IV or V, due to clinical- histological correlation or renal biopsy; with cumulative doses of $9.44 \pm 4.22$ grams. Fifteen patients received IV cyclophosphamide and methylprednisolone. Among patients using cyclophosphamide, $60(80 \%)$ completed remission induction period and, then, received $2-3 \mathrm{mg} / \mathrm{kg} /$ day doses of azathioprine for maintenance treatment. Fifteen patients discontinued cyclophosphamide due to intolerance or adverse effects, such as gastrointestinal effects (nausea and vomit in 8 patients), infections (5 patients) and severe leucopenia $\left(<2000\right.$ cells $/ \mathrm{mm}^{3}$ in 4 individuals). In case of cyclophosphamide discontinuation during induction phase, azathioprine was administered in 10 patients, and mycophenolate mofetil was used in 5 cases, in a $2 \mathrm{~g}$ /day doses, taken twice a day. Most patients with cutaneous and articular manifestations (85\%) were taking antimalarials $(6 \mathrm{mg} / \mathrm{kg} /$ day of hydroxychloroquine or $4 \mathrm{mg} / \mathrm{kg}$ /day of chloroquine diphosphate).

Renal variables analyzed were: Serum creatinine, 24-hour proteinuria, changes in urinary sediment and fractional complement. Comparing such variable results at the beginning and after 12 months of follow-up, it was found that all patients showed significant reduction in the 24-hour urinary protein level, improvement in the urinary sediment and increase in the fractional values of the complement (Table 4). During followup, there was a trend to increment serum creatinine, without, however, reaching statistic significance.

Complete remission frequency at 12 month was $72.7 \%$, and at 24 months, $85.7 \%(p=0,013)$. Partial remission was achieved in $27.3 \%$ of patients at 12 months, and $14.3 \%$ at 24 months (Figure 1). Comparison among subgroups with complete and partial remission at 12 months and the baseline parameters of SLE and nephritis showed that no variable had correlation with therapeutic response (Table 5). Renal flare during nephritis treatment was observed in $40 \%$ of cases, most of them (80\%) during maintenance phase. In such cases, glucorticoid and immunosuppressive doses were optimized, according to doses intervals described above.

Male patients presented a lower rate of complete remission when compared with female at 12 months ( $45.5 \%$ versus $81.6 \%$, respectively, $p=0.007$ ), which could suggest a less favorable outcome of lupus nephritis among men of this population. (Figure 2). As may be verified by the presented data, men who did not achieve complete remission at 12 months did accomplish partial response to the established treatment.

Comparative analysis of "clinical class IV", "histological class IV", and initial variables of nephritis and SLE, showed that there was no statistical difference among SLEDAI values, serum creatinine and 24-hour proteinuria, as well as in the urinary sediment, the fraction values of complement and the cumulative doses of cyclophosphamide in both groups (Table 6). The rate of complete remission was higher in patients with "clinical class IV" than in those with "histological class IV". (Figure 3)

\section{DISCUSSION}

In this study, we report the evolution of 100 patients with lupus nephritis followed up for two years. The treatment regimen of our centre used IV cyclophosphamide as a first-choice immunosuppressive agent for induction of class III, IV or V nephritis remission. Other agents like azathioprine or MMF were used for nephritis remission-maintenance treatment after 6-9 months IV cyclophosphamide, or in case of failure or intolerance to the previously described regimen. According to Contreras et 
Table 2

Analysis of 100 patients with lupus nephritis followed up for 2 years. Demographic data.

\begin{tabular}{lc}
\hline $\begin{array}{l}\text { Age at SLE diagnosis in years } \\
(\mathrm{M} \pm \mathrm{SD})\end{array}$ & $\begin{array}{c}24.71 \pm 10.14 \\
(5-46)\end{array}$ \\
$\begin{array}{l}\text { Age at nephritis diagnosis in years } \\
(\mathrm{M} \pm \mathrm{SD})\end{array}$ & $\begin{array}{c}\text { (5.78 } \\
(5-53)\end{array}$ \\
$\begin{array}{l}\text { Interval between SLE diagnosis and } \\
\text { nephritis diagnosis in years } \\
(\mathrm{M} \pm \mathrm{SD})\end{array}$ & $2.32 \pm 4.70$ \\
Women $(\%)$ & $(0-24)$ \\
Race $(\%)$ & $88(88 \%)$ \\
White & \\
Others & $57(57 \%)$ \\
Non registered & $29(29 \%)$ \\
\hline
\end{tabular}

SLE = Systemic Lupus Erythematosus; $\mathrm{M}$ = mean; $\mathrm{SD}$ = standard deviation.

Table 3

Initial clinical and laboratory data of 100 patients with lupus nephritis

\begin{tabular}{|c|c|}
\hline $\begin{array}{l}\text { Creatinine in } \mathrm{mg} / \mathrm{dL} \\
(\mathrm{M} \pm \mathrm{SD} / \text { variation })\end{array}$ & $\begin{array}{l}1.02 \pm 0.49 \\
(0.4-3.6)\end{array}$ \\
\hline $\begin{array}{l}\text { Proteinuria in } g / 24 h \\
(M \pm S D / \text { variation })\end{array}$ & $\begin{array}{c}2.57 \pm 2.39 \\
(0-10.3)\end{array}$ \\
\hline SAH (\%) & $37(37 \%)$ \\
\hline Anti-dsDNA positivity (\%) & $66(66 \%)$ \\
\hline
\end{tabular}

$\mathrm{M}=$ mean; $\mathrm{SD}$ = standard deviation; $\mathrm{SAH}=$ Systemic Arterial Hypertension; Anti-dsDNA = double-stranded-dsDNA.

\section{Table 4}

Comparison of variables related to nephritis at baseline $\left(\mathrm{T}_{0}\right)$ and after 12 months $\left(\mathrm{T}_{12}\right)$ in 100 patients with lupus nephritis

\begin{tabular}{lccc}
\hline & $\mathrm{T}_{0}$ & $\mathrm{~T}_{12}$ & $P^{*}$ \\
\hline $\begin{array}{l}\text { Creatinine in } \mathrm{mg} / \mathrm{dL} \\
(\mathrm{M} \pm \mathrm{SD})\end{array}$ & $0.99 \pm 0.42$ & $1.03 \pm 0.94$ & 0.663 \\
$\begin{array}{l}\text { Proteinuria in } \mathrm{g} / 24 \mathrm{~h} \\
(\mathrm{M} \pm \mathrm{SD})\end{array}$ & $2.58 \pm 2.42$ & $1.26 \pm 1.83$ & 0.000 \\
$\begin{array}{l}\text { Hematuria in cell } \mathrm{p} / \mathrm{f} \\
(\mathrm{M} \pm \mathrm{SD})\end{array}$ & $22.99 \pm 23.42$ & $13.06 \pm 18.64$ & 0.000 \\
\hline $\begin{array}{l}\text { Leucocituria in cell } \mathrm{p} / \mathrm{f} \\
(\mathrm{M} \pm \mathrm{SD})\end{array}$ & $14.61 \pm 16.77$ & $10.10 \pm 15.34$ & 0.037 \\
$\mathrm{CH} 50(\mathrm{M} \pm \mathrm{SD})$ & $172.50 \pm$ & $190.67 \pm$ & 0.031 \\
\hline $\mathrm{C} 4(\mathrm{M} \pm \mathrm{SD})$ & $16.96 \pm 8.04$ & $21.48 \pm 9.86$ & 0.024 \\
$\mathrm{C} 3(\mathrm{M} \pm \mathrm{SD})$ & $82.44 \pm 39.36$ & $100.12 \pm$ & 0.026 \\
\hline
\end{tabular}

${ }^{*} t$ de student test

$\mathrm{M}=$ mean; $\mathrm{DP}=$ standard deviation; $\mathrm{Cel} \mathrm{p} / \mathrm{f}=$ cells per field;

$\mathrm{CH} 50=$ total serum hemolytic complement; $\mathrm{C} 4=$ complement $4 ; \mathrm{C} 3=$ complement 3

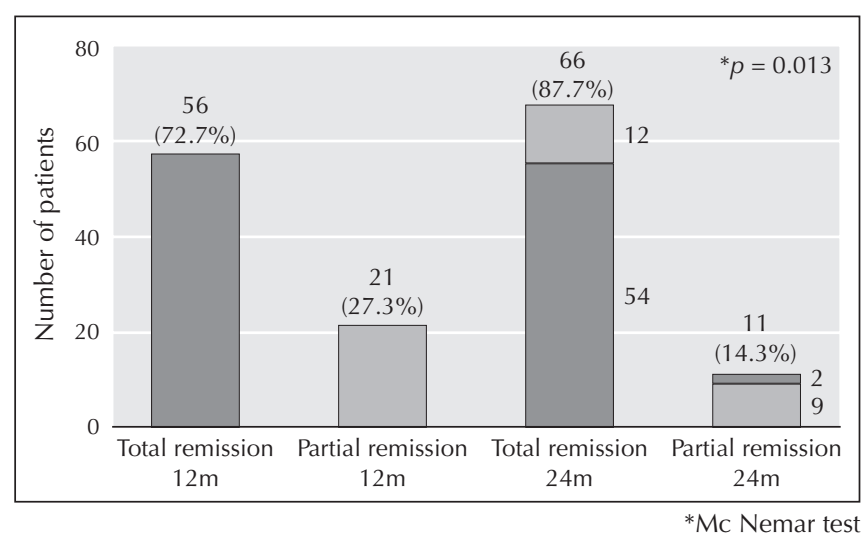

Figure 1. Comparison among subgroups with total and partial remission at 12 months and 24 months of follow-up $(n=77)$.

Table 5

Comparison among subgroups with total and partial remission at 12 months and SLE and nephritis variables at baseline: Prognostic factors.

\begin{tabular}{|c|c|c|c|}
\hline & $\begin{array}{l}\text { Total Remission } \\
\quad(n=76)\end{array}$ & $\begin{array}{l}\text { Parcial Remission } \\
\quad(\mathrm{n}=22)\end{array}$ & $* p$ \\
\hline Age in years $(M \pm S D)$ & $26.57 \pm 11.33$ & $27.90 \pm 9.04$ & 0.570 \\
\hline $\operatorname{SLEDAI}(M \pm S D)$ & $16.17 \pm 6.57$ & $15.77 \pm 6.47$ & 0.802 \\
\hline $\begin{array}{l}\text { Creatinine in } \mathrm{mg} / \mathrm{dL} \\
(\mathrm{M} \pm \mathrm{DP})\end{array}$ & $0.94 \pm 0.86$ & $1.13 \pm 0.55$ & 0.147 \\
\hline $\begin{array}{l}\text { Proteinuria in } g / 24 h \\
(M \pm S D)\end{array}$ & $2.31 \pm 2.19$ & $3.49 \pm 2.94$ & 0.093 \\
\hline $\begin{array}{l}\text { Hematuria in cel } p / f \\
(M \pm S D)\end{array}$ & $21.61 \pm 22.38$ & $27.77 \pm 26.73$ & 0.332 \\
\hline $\begin{array}{l}\text { Leucocituria in cel } p / f \\
(M \pm S D)\end{array}$ & $12.20 \pm 13.35$ & $22.95 \pm 23.87$ & 0.064 \\
\hline $\mathrm{CH} 50(\mathrm{M} \pm \mathrm{SD})$ & $163.97 \pm 119.62$ & $140.75 \pm 111.77$ & 0.614 \\
\hline $\mathrm{C} 3(\mathrm{M} \pm \mathrm{SD})$ & $78.39 \pm 34.61$ & $84.15 \pm 42.68$ & 0.663 \\
\hline $\mathrm{C} 4(\mathrm{M} \pm \mathrm{SD})$ & $16.28 \pm 8.63$ & $16.08 \pm 8.01$ & 0.938 \\
\hline $\begin{array}{l}\text { Cumulative dosis of } \\
\text { cyclophosphamide in } g \\
(\mathrm{M} \pm \mathrm{SD})\end{array}$ & $9.33 \pm 3.84$ & $10.27 \pm 4.77$ & 0.42 \\
\hline \multicolumn{4}{|l|}{${ }^{*} t$ de student test } \\
\hline \multicolumn{4}{|c|}{$\begin{array}{l}\text { SLE = Systemic Lupus Erythematosus, } \mathrm{M}=\text { mean, } \mathrm{SD}=\text { standard deviation; } \\
\text { SLEDAI = Systemic Lupus Erythematosus Disease Activity Index; } \\
\text { Cel p/f = cells per field; CH50 = total serum hemolytic complement; } \\
\text { C4 = complement 4; = C3 complement } 3 .\end{array}$} \\
\hline
\end{tabular}

$a l .^{6}$, patients with diffuse proliferative lupus nephritis who had received intravenous cyclophosphamide for a short period of time, followed by MMF or azathioprine maintenance therapy, demonstrate more effective results and more safety than the prolonged use of cyclophosphamide. Houssiau corroborated that IV cyclophosphamide is the only therapy able to reduce 


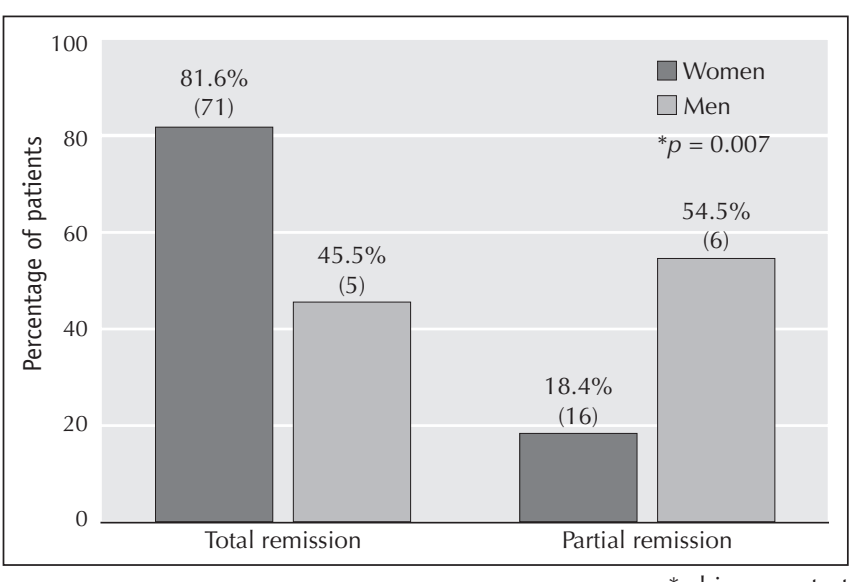

* chi-square test

Figure 2. Comparison among men and women subgroups referring to total and partial remission at 12 months.

Table 6

Comparison among "clinical class IV" and

"histological class IV" subgroups

\begin{tabular}{|c|c|c|c|}
\hline & \multicolumn{2}{|c|}{ Class IV } & \multirow[b]{2}{*}{$* p$} \\
\hline & $\begin{array}{l}\text { Histological } \\
\quad(n=18)\end{array}$ & $\begin{array}{l}\text { Clinical } \\
(n=23)\end{array}$ & \\
\hline $\operatorname{SLEDAI}(M \pm S D)$ & $16.22 \pm 5.26$ & $17.83 \pm 5.67$ & 0.356 \\
\hline $\begin{array}{l}\text { Creatinine in } \mathrm{mg} / \mathrm{dL} \\
(M \pm S D)\end{array}$ & $1.13 \pm 0.33$ & $1.09 \pm 0.37$ & 0.707 \\
\hline $\begin{array}{l}\text { Proteinuria in } g / 24 h \\
(M \pm S D)\end{array}$ & $2.67 \pm 2.12$ & $3.37 \pm 2.70$ & 0.360 \\
\hline $\begin{array}{l}\text { Hematuria in cel } p / f \\
(M \pm S D)\end{array}$ & $37.33 \pm 25.22$ & $34.96 \pm 26.33$ & 0.771 \\
\hline $\begin{array}{l}\text { Leucocituria in cel } p / f \\
(M \pm S D)\end{array}$ & $15.61 \pm 12.77$ & $19.78 \pm 23.44$ & 0.472 \\
\hline $\mathrm{CH} 50(\mathrm{M} \pm \mathrm{SD})$ & $135.71 \pm 119.62$ & $124.22 \pm 83.65$ & 0.833 \\
\hline $\mathrm{C} 3(\mathrm{M} \pm \mathrm{SD})$ & $86.76 \pm 46.82$ & $58.53 \pm 19.17$ & 0.117 \\
\hline $\mathrm{C} 4(\mathrm{M} \pm \mathrm{SD})$ & $15.00 \pm 6.61$ & $13.07 \pm 6.90$ & 0.511 \\
\hline $\begin{array}{l}\text { Cumulative dosis of } \\
\text { cyclophosphamide in } g \\
(\mathrm{M} \pm \mathrm{SD})\end{array}$ & $8.72 \pm 3.79$ & $10.31 \pm 4.48$ & 0.227 \\
\hline \multicolumn{4}{|l|}{$t$ de student test } \\
\hline $\begin{array}{l}\mathrm{M}=\text { mean, } \mathrm{SD}=\text { standard deviati } \\
\text { Activity Index; Cel } \mathrm{p} / \mathrm{f}=\text { cells } \\
\mathrm{C} 4=\text { complement } 4 ;=\mathrm{C} 3 \text { comple }\end{array}$ & $\begin{array}{l}\mathrm{Al}=\text { Systemic } \mathrm{Lupl} \\
\mathrm{CH} 50=\text { total serum he }\end{array}$ & $\begin{array}{l}\text { us Erythematosus } \\
\text { emolytic complement; }\end{array}$ & \\
\hline
\end{tabular}

development into ESRD in these patients based in studies with long period of follow-up. ${ }^{7}$

Our data demonstrated satisfactory outcomes in all patients at the end of the 12-month follow-up. Among renal variables analyzed, there was a significant reduction in the 24-hour urinary protein level, improvement in the urinary sediment and increase in the fractional values of the complement ( C3, C4,

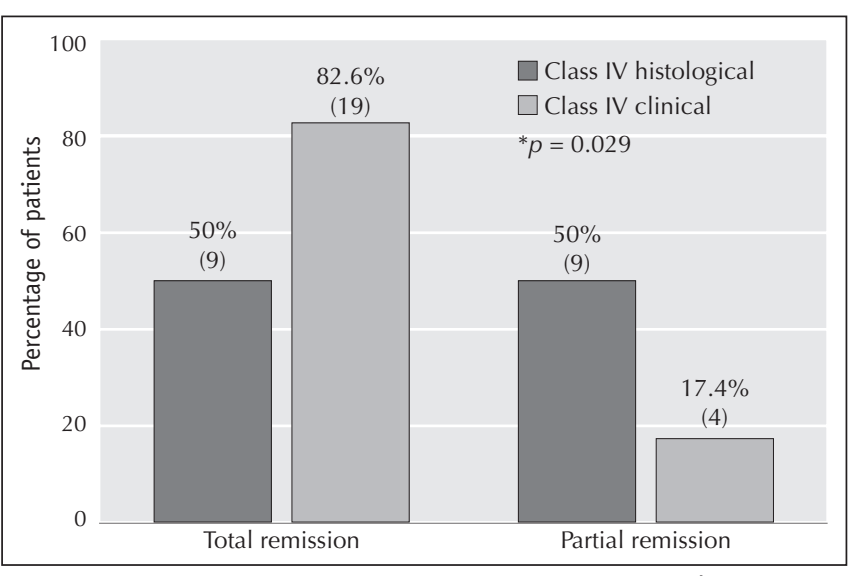

* chi-square test

Figure 3. Comparison among subgroups with "clinical class IV" and "histological class IV" total and partial remission at 12 months of follow-up.

CH50). The frequency of complete remission at 12 months was $72.7 \%$ and, at 24 months, $85.7 \%(p=0,013)$. According to Urowitz et al., patients with active lupus nephritis treated with cyclophosphamide or other immunosuppressive achieve complete remission in approximately $70 \%$ of cases. ${ }^{8}$

According to our data, no patient presented clinical and laboratory criteria for lack of therapeutic response by the end of 2-year of follow-up. However, these data should be carefully interpreted, since, during the study period, there was recurrence of active renal disease (flare) in approximately $40 \%$ of the patients, but after treatment optimization (increment of corticosteroids and/or immunosuppressive doses) these patients responded in a satisfactory way.

Comparison among subgroups with complete and partial remission at 12 months and the baseline variables of SLE and nephritis showed that no variable had correlation with therapeutic response. Previous studies demonstrated that variables like age, proteinuria, hematuria and arterial hypertension at diagnosis time had a questionable prognostic value in lupus nephritis outcome. ${ }^{9,10,11,12}$ Other authors concluded in their studies that high creatinine and hypoalbuminaemia represented factors of predicting worst renal prognosis. ${ }^{13,14}$ In a study published in Brazil, ${ }^{15}$ it was prooved that patients with SLE onset after 16 years old, the worse outcomes had occurred in those with nephritis, revealed clinically at the beginning or in the evolution of the disease, with arterial hypertension in the beginning, with positive anti-DNA and those with cyclophosphamide use. Possible explanations for these contradictory facts derive mainly from methodological differences of the series: criteria of patient inclusion and selection, retrospective 
studies, follow-up periods, treatment regimen, clinical outcome definition, sample size and others.

Male patients presented a lower rate of complete remission compared to female at 12 months, what could suggest worse prognosis of lupus nephritis among men in our population. This data differ from that in literature, where gender has no prognostic value in lupus nephritis outcome. ${ }^{16}$

Despite the small number of patients studied, and the controversies of renal biopsy, the rates of complete remission was higher in patients with "clinical class IV" than in those with "histological class IV" at 12 months. A balance between potential gain of information that can guide a therapeutic approach and complications associated with invasive procedure must always be considered before recommending a renal biopsy. Previous studies demonstrated that clinical and laboratory parameters are able to predict with accuracy the lupus nephritis prognosis, and biopsy is not required in every case with renal involvement. ${ }^{17,18}$ Other studies defend that histological data provide additional information to laboratory parameters and may guide, in a more accurate way, therapeutic selection. ${ }^{19}$ As may be noticed, this topic remains controvertial. In our experience, renal biopsy is individual and assigned, mainly, in the following situations: Question about clinical and histological correlation, presence of nephrotic syndrome or, failure to cyclophosphamide induction therapy. For this study, the frequency of complete remission at 12 months was significantly higher in patients with "clinical class IV", without the need for additional information by histological examination. In our data, renal variables and SLE, as well as cumulative cyclophosphamide doses were not different among patients with "clinical class IV" and "histological class IV", that is, with renal biopsy.

In conclusion, in this study, renal biopsy to determine the histological subtype did not influenced on therapeutic response in lupus nephritis patients during two years of follow-up. Our data demonstrated that clinical and histological correlation may help therapeutic decision of these patients, leaving renal biopsy only for special situations.

\section{REFERÊNCIAS BIBLIOGRÁFICAS}

\section{REFERENCES}

1. Appel GB, Valeri A. The course and treatment of lupus nephritis. Ann Rev Med 1994;45:525-37.

2. Cervera R, Khamashta MA, Font J, Sebastiani GD, Gil A, Lavilla P, et al. Morbidity and mortality in systemic lupus erythematous during a 10-year period: a comparison of early and late manifestations in a cohort of 1,000 patients. Medicine. 2003;82:299-308.
3. Steinberg AD, Steinberg SC. Long-term preservation of renal functions in patients with lupus nephritis receiving treatment that includes cyclophosphamide versus those treated with prednisone only. Arthritis Rheum. 1991;34:945-50.

4. Esdaile JM, Federgreen W, Quintal H, Suissa A, Hayslett JP, Kashgarian M. Predictors of one year outcome in lupus nephritis: The importance of renal biopsy. Q J Med. 1991;81:907-18

5. Boumpas DT, Balow JE. Outcome criteria for lupus nephritis trials: a critical overview. Lupus. 1998;7:622-9.

6. Contreras G, Pardo V, Leclercq B, Lenz O, Tozman E, O'Nan P, et al. Sequential therapies for proliferative lupus nephritis. 2004 ;350: 971-80.

7. Houssiau FA, Vasconcelos C, D'Cruz D, Sebastiani GD, Garrido Ed Ede R, Danieli MG, et al. Immunosuppressive therapy in lupus nephritis: the Euro-Lupus Nephritis Trial, a randomized trial of low-dose versus high-dose intravenous cyclophosphamide. Arthritis Rheum. 2002;46:2121-31.

8. Urowitz MB, Ibañez D, Ali Y, Gladman DD. Outcomes in patients with active lupus nephritis requiring immunosupressives who never received cyclophosphamide. J Rheumatol. 2007;34:1491-96.

9. Donadio Jr JV, Hart GM, Bergstralh EJ. Prognostic determinants in lupus nephritis: a long-term clinicopathologic study. Lupus. 1995;4: 109-15.

10. Bakir AA, Levy PS, Dunea G. The prognosis of lupus nephritis in African-Americans: A retrospective analysis. Am J Kidney Dis. 1994;24:159-71.

11. Appel GB, Cohen DJ, Pirani CL, Meltzer JL, Estes D. Longterm follow-up of patients with lupus nephritis. A study based on the classification of the World Health Organization. Am J Med. 1987;83:877-85.

12. Baqi N, Moazami S, Singh A, Ahmad H, Balachandra S, Tejani A. Lupus nephritis in children: A longitudinal study of prognostic factors and therapy. J Am Soc Nephrol. 1996;7:924-9.

13. Seedat YK, Parag KB, Ramsaroop R. Systemic lupus erythematous and renal involvement. A South African experience. Nephron. 1994;66:426-30.

14. Villas Boas ML, Nakayama E, Carvalho, MFC, Delfino Filho J, Neiva SLA, Habermann F, et al. Lupus eritematoso sistêmico: estudo de 48 pacientes com ênfase no comprometimento renal. Rev Ass Med Bras. 1988;34:165-73.

15. Costallat LTL, Appenzeller S, Marini R. Evolução e fatores prognósticos do lúpus eritematoso sistêmico em relação com a idade de início. Rev Bras Reumatol. 2002;42(2):91-8.

16. Leaker B, Fairley KF, Dowling J, Kincaid-Smith P. Lupus nephritis: clinical and pathological correlation. Q J Med. 1987;62:163-79.

17. Whiting-O'Keefe Q, Henke JE, Shearn MA, Hopper J Jr, Biava CG, Epstein WV. The information content from renal biopsy in systemic lupus erythematosus. Ann Intern Med. 1982;96(part 1):718-23.

18. Austin HA, Muenz LR, Joyce KM, Antonovych TA, Kullick ME, Klippel JH, et al. Prognostic factors in lupus nephritis. Contribution of renal histologic data. Am J Med. 1983;75:382-91.

19. Moroni G, Pasquali S, Quaglini S, Banfi G, Casanova S, Maccario $\mathrm{M}$, et al. Clinical and prognostic value of serial renal biopsies in lupus nephritis. Am J Kidney Dis. 1999;34:530-9. 